\title{
Regeneration of Photopigment Is Enhanced in Mouse Cone Photoreceptors Expressing RPE65 Protein
}

\author{
Peter H. Tang, ${ }^{1}$ Lee Wheless, ${ }^{2}$ and Rosalie K. Crouch ${ }^{1,3}$ \\ Departments of ${ }^{1}$ Neuroscience, ${ }^{2}$ Medicine-Division of Biostatistics and Epidemiology, and ${ }^{3}$ Ophthalmology, Medical University of South Carolina, \\ Charleston, South Carolina 29425
}

As cone photoreceptors mediate vision in bright light, their photopigments are bleached at a rapid rate and require substantial recycling of the chromophore 11-cis-retinal (RAL) for continued function. The retinal pigment epithelium (RPE) supplies 11-cis-RAL to both rod and cone photoreceptors; however, stringent demands imposed by the function of cones in bright light exceed the output from this source. Recent evidence has suggested that cones may be able to satisfy this demand through privileged access to an additional source of chromophore located within the inner retina. In this study, we demonstrate that the protein RPE65, previously identified in RPE as the isomerohydrolase of the RPE-retinal visual cycle, is found within cones of the rod-dominant mouse retina, and the level of RPE65 in cones is inversely related to the level in the RPE. The light sensitivity of cone ERGs of $B A L B / c$ mice, which had an undetectable level of cone RPE65, was enhanced by approximately threefold with administration of exogenous chromophore, indicating that the cones of these animals are chromophore deficient. This enhancement with chromophore administration was not observed in $C 57 B L / 6$ mice, whose cones contain RPE65. These results demonstrate that RPE65 within cones may be essential for the efficient regeneration of cone photopigments under bright-light conditions.

\section{Introduction}

Vitamin A aldehyde, 11-cis-retinal (RAL), acts as the chromophore within rod and cone photoreceptors by covalently binding opsin G-protein-coupled receptors to form photopigment. When photons are absorbed by the photopigment, 11-cisRAL is isomerized to the all-trans conformation, triggering the phototransduction cascade for propagating neuronal signals to the visual cortex. To replenish photopigments, all-trans RAL is recycled back to 11-cis-RAL through a series of enzymatic steps known as the visual cycle. The most well known pathway is a cycling of vitamin A derivatives (retinoids) from photoreceptors through the retinal pigment epithelium (RPE), known as the RPE-retinal visual cycle (Travis et al., 2007). Within the $\mathrm{RPE}$, the protein RPE65 acts as the isomerohydrolase to convert all-trans retinoids back to 11-cis-forms (Jin et al., 2005;

Received Jan. 11, 2011; revised May 19, 2011; accepted May 30, 2011.

Author contributions: P.H.T. and R.K.C. designed research; P.H.T. performed research; P.H.T. contributed unpublished reagents/analytic tools; P.H.T., L.W., and R.K.C. analyzed data; P.H.T., L.W., and R.K.C. wrote the paper.

The authors declare no competing financial interests.

This study was supported in part by National Institutes of Health Grants R01 EY04939 (R.K.C.) and C06 RR015455 [Medical University of South Carolina (MUSC)], Foundation Fighting Blindness (R.K.C.), an unrestricted grant (Department of Ophthalmology, MUSC) from Research to Prevent Blindness (RPB), a RPB Senior Scientific Investigator Award (R.K.C.), and a RPB Medical Student Research Fellowship (P.H.T.). We thank Debra Thompson (University of Michigan, Ann Arbor, MI) for generously supplying the 8B11 anti-RPE65 antibody, for extensive discussion of the experiments, for undertaking experiments with our antibodies in her laboratory, and for comments on a previous version of this manuscript. We also thank Vladimir Kefalov (Washington University at St. Louis, St. Louis, M0), Venkat Ramshesh, Joseph Palatinus, Patrice Goletz, Jie Fan, Luanna Bartholomew, Ryan Parker, Yiannis Koutalos, Masahiro Kono, and Bärbel Rohrer (Medical University of South Carolina, Charleston, SC) for technical assistance, discussions, and manuscript revisions.

Correspondence should be addressed to Rosalie K. Crouch, Department of Ophthalmology, Medical University of South Carolina, 167 Ashley Avenue, Charleston, SC 29425. E-mail: crouchrk@musc.edu.

DOI:10.1523/JNEUROSCI.0182-11.2011

Copyright $\odot 2011$ the authors $\quad 0270-6474 / 11 / 3110403-09 \$ 15.00 / 0$
Moiseyev et al., 2005; Redmond et al., 2005). Once 11-cis-RAL is regenerated, it is shuttled to photoreceptors to reform photopigment. The levels of RPE65 in the RPE have been shown to vary in different strains of wild-type mice (Lyubarsky et al., 2005).

Numerous studies have suggested that the mechanisms by which rods and cones obtain chromophore may differ (Wang and Kefalov, 2011). Cones function in daylight to mediate high-resolution color vision, and their rates of sensitivity recovery (Perry and McNaughton, 1991) and photopigment regeneration (Schnapf et al., 1990) are greater than that of rods (Baylor et al., 1979, 1984), which function in low-light conditions. Concurrently, the rate of photopigment regeneration that is necessary for sustaining cone function in bright light exceeds the capabilities of the RPE to recycle chromophore (Mata et al., 2002).

A second visual cycle may exist to supplement cones with chromophore (Muniz et al., 2007). This alternate pathway is localized entirely within the retina (intraretinal visual cycle) and functions in parallel with the RPE-retinal visual cycle. Cones can use vitamin A alcohol, 11-cis-retinol (ROL), as a source of chromophore, presumably by oxidizing 11-cis-ROL to 11-cis-RAL through an uncharacterized dehydrogenase activity (Jones et al., 1989; Ala-Laurila et al., 2009). Rods lack this capability, and thus 11-cis-ROL is not a viable substrate for these photoreceptors (Kono et al., 2008). Müller cells may contribute to the intraretinal visual cycle by producing 11-cis-ROL from all-trans ROL (Das et al., 1992; Wang and Kefalov, 2009; Wang et al., 2009); however, the mechanism is currently not known.

There is evidence to support the existence of RPE65 within cones, in addition to the RPE. The detection of Rpe65 mRNA has 

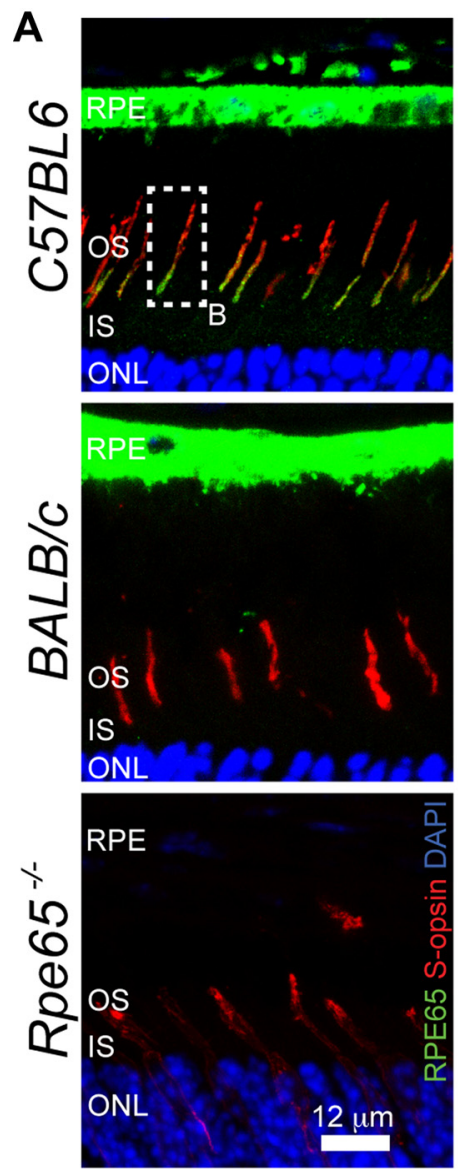

D

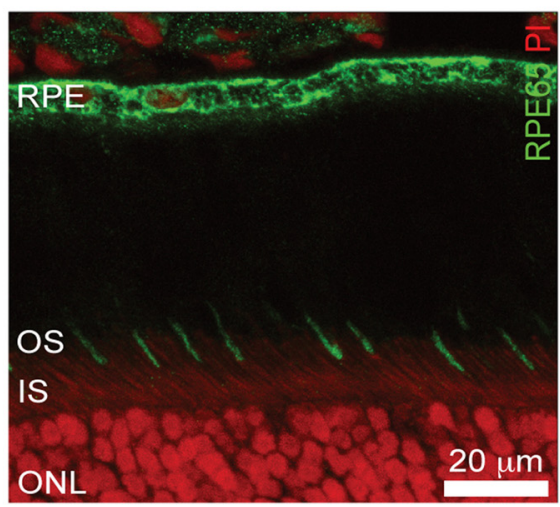

B
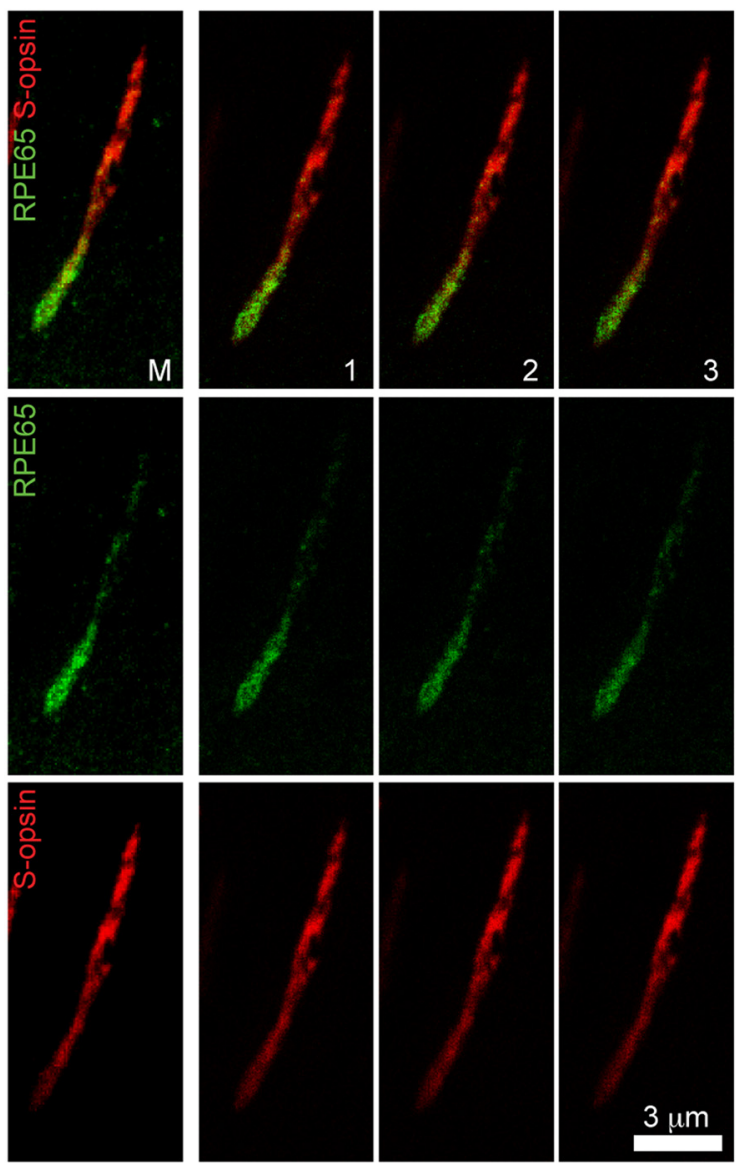

E

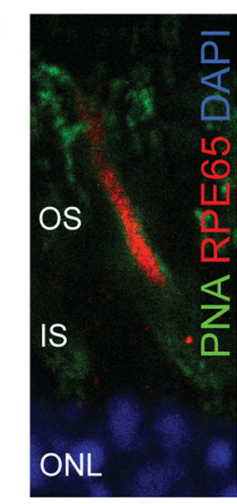

C

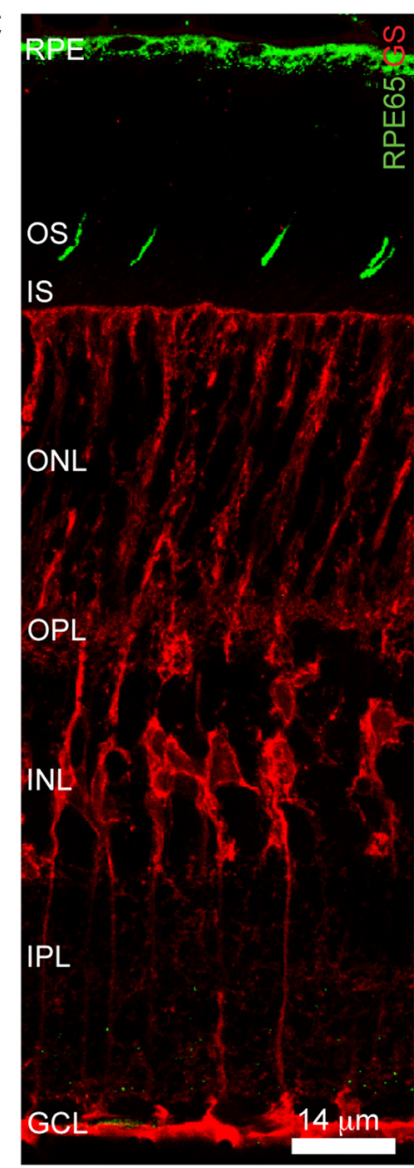

Figure 1. Localization of RPE65 protein within the cone OS. A, Frozen sections prepared from 4-week-old C57BL/6 (top) and BALB/C (middle) eyecups and 2-week-old Rpe65 ${ }^{-/-}$eyecups (bottom) were costained for RPE65 (PETLET antibody), S-opsin, and nuclei (DAPI). B, Analysis of sequential single-plane images acquired as a $z$-series (1-3) and the merged image (M) from a single-cone 0S is displayed, showing the distribution of RPE65.C, C57BL/6 eyecup section costained for RPE65 and GS, showing the absence of RPE65 in the Müller glia. D, Visualization of IS in C57BL/6 mouse eyecup using propidium iodide (PI) allows for additional analysis of RPE65 localization within the cone, demonstrating that the majority of RPE65 is localized at the base of the cone $0 S$. $E$, FITC-conjugated PNA was used to counterstain the glycoprotein sheath surrounding C57BL/6 mouse cone OS and IS, and a single-plane image was acquired to show the glycoprotein sheath surrounding RPE65 immunostaining within the OS. ONL, Outer nuclear layer; OPL, outer plexiform layer; INL, inner nuclear layer; IPL, inner plexiform layer; GCL, ganglion cell layer.

been reported in isolated salamander cones (Ma et al., 1998), and immunohistochemistry (IHC) analysis of retinas from a variety of animal species confirmed the presence of the protein (Znoiko et al., 2002). These findings have generated debate because independent studies failed to reproduce the results (Seeliger et al., 2001; Hemati et al., 2005). The current study was undertaken to reevaluate the localization of RPE65 protein within cones of different mouse strains/lines and to determine whether there is a relationship between the presence of RPE65 within cones and their function.

\section{Materials and Methods}

Animals. Experimental procedures and animal care protocols adhered to the Association for Research in Vision and Ophthalmology Statement for the Use of Animals in Ophthalmic and Vision Research and were approved by the Institutional Animal Care and Use Committee of the Medical University of South Carolina. C57BL/6 mice were obtained from The Jackson Laboratory. BALB/c, 129Sv, and albino C57BL/6 (C57BL/6-Tyr ${ }^{c}$ / $\mathrm{BrdCrHsd}$ ) mice were obtained from Harlan Laboratories. Rpe65 ${ }^{-1-}$ mice were a generous gift from T. Michael Redmond (National Eye Institute, Bethesda, MD). All lines of mice used for experiments were F1 

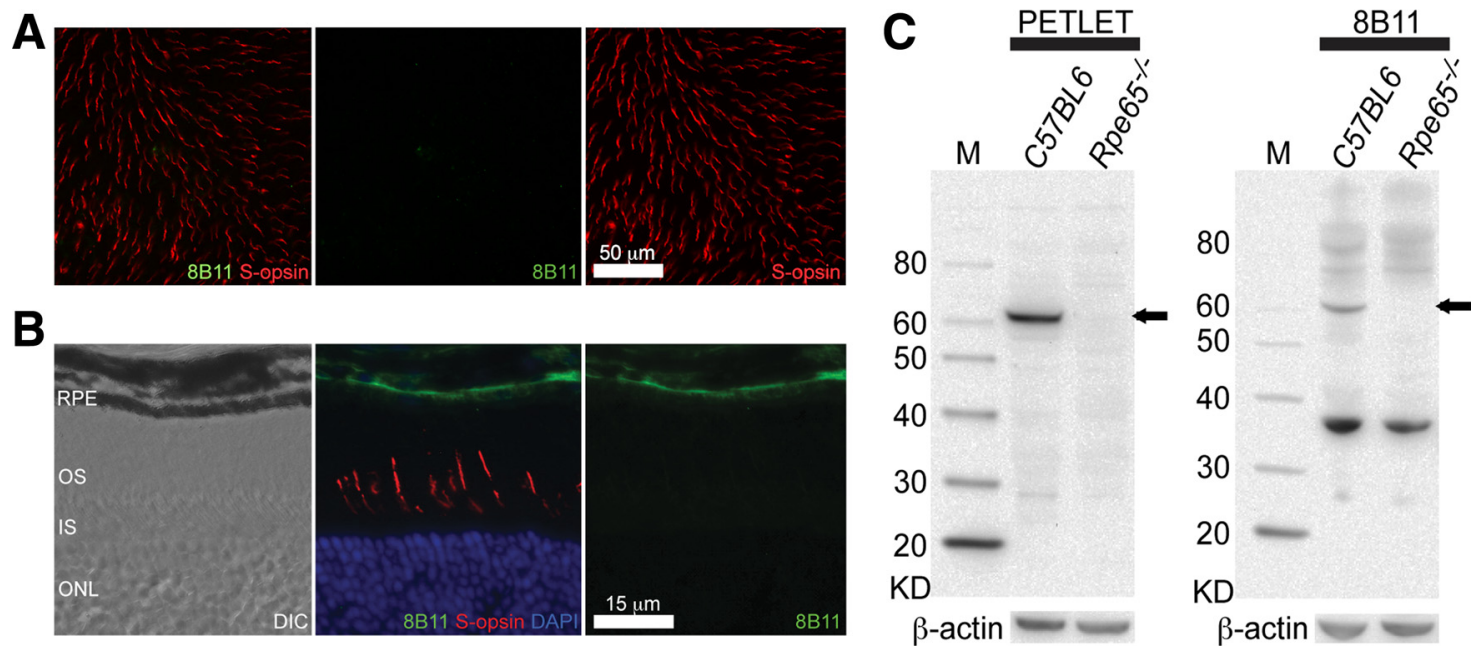

Figure 2. Specificity of anti-RPE65 antibodies. Immunostaining of the 8B11 anti-RPE65 antibody was evaluated with 4-week-old C57BL/6 retinas and eyecups. A, The 8B11 antibody failed to detect RPE65 within cones of the flat-mounted retina. $\boldsymbol{B}$, Evaluation of eyecup sections provided confirmation, with $8 B 11$ immunostaining detected only within the RPE. $\boldsymbol{C}$, Immunoblot analysis of whole eyecup homogenates prepared from 2-week-old C57BL/6 and Rpe65 ${ }^{-1-}$ mice with the PETLET antibody showed detection of a $61 \mathrm{kDa}$ major band corresponding to RPE65 protein in the C57BL/6 sample but not in the Rpe65 ${ }^{-1-}$ sample. The $8 \mathrm{~B} 11$ antibody also detected RPE65 protein in the C57BL/6 sample but none in the Rpe65 ${ }^{-1-}$ sample. Blots were stripped and reprobed for $\beta$-actin, shown as insets. Arrows represent location of RPE65 band; M, marker; DIC, differential interference contrast; $0 \mathrm{NL}$, outer nuclear layer.

progeny generated from mating the various strains, and members of either sex were evaluated.

Anti-RPE65-specific primary antibodies. Two anti-RPE65-specific antibodies were used for the current study. The PETLET polyclonal rabbit antibody has been described previously to detect immunoreactive RPE65 protein (Znoiko et al., 2002) and was used at a concentration of $2 \mu \mathrm{g} / \mathrm{ml}$ for IHC analysis and $0.2 \mu \mathrm{g} / \mathrm{ml}$ for immunoblot analysis. The $8 \mathrm{~B} 11$ monoclonal mouse antibody was a generous gift from Debra A. Thompson (University of Michigan, Ann Arbor, MI) (Hemati et al., 2005) and was used at a dilution of $2 \mu \mathrm{g} / \mathrm{ml}$ for IHC analysis and $0.2 \mu \mathrm{g} / \mathrm{ml}$ for immunoblot analysis.

Other primary antibodies. The antibody raised against short-wavelength (S-) cone opsin and used for IHC was obtained from Santa Cruz Biotechnology (catalog \#sc-14363) and was used at a concentration of $0.4 \mu \mathrm{g} / \mathrm{ml}$. The anti-S-opsin (catalog \#AB5407) and anti-mid-wavelength (M-) opsin (catalog \#AB5405) antibodies for immunoblot analysis were obtained from Millipore Bioscience Research Reagents and were both used at a concentration of $0.1 \mu \mathrm{g} / \mathrm{ml}$. The anti- $\beta$-actin antibody for immunoblot analysis was obtained from Sigma-Aldrich (catalog \#A1978) and was used at a concentration of $0.5 \mu \mathrm{g} / \mathrm{ml}$. FITC-conjugated peanut agglutinin (PNA lectin) was obtained from Sigma-Aldrich (catalog \#L7381) and was used at a concentration of $2 \mu \mathrm{g} / \mathrm{ml}$ for IHC analysis. The antibody raised against glutamine synthetase (GS) was obtained from BD Biosciences (catalog \#610517) and was used at a concentration of $0.1 \mu \mathrm{g} / \mathrm{ml}$.

Immunoblot analysis. Procedures were modified from methods described previously (Tang et al., 2010). To prepare mouse whole-eyecup homogenates, eyes were enucleated and dissected to remove cornea and lens. The peroxidase-conjugated secondary antibodies used were goat anti-rabbit IgG ( $1 \mathrm{ng} / \mathrm{ml}$ concentration; Vector Laboratories) and horse anti-mouse IgG ( $1 \mathrm{ng} / \mathrm{ml}$ concentration; Vector Laboratories).

IHC analysis of flat-mounted retina. Procedures have been described previously (Tang et al., 2010). Retinas were incubated with appropriate primary antibodies overnight at $4^{\circ} \mathrm{C}$, washed with $\mathrm{PBS}[2 \times, 10 \mathrm{~min}$, room temperature (RT)], and then incubated with Alexa Fluor- 488 and -594 secondary antibodies $(0.4 \mu \mathrm{g} / \mathrm{ml}$ concentration; Invitrogen $)$ for $2 \mathrm{~h}$ at RT. To calculate cone density, cones were counted based on S-opsin staining within the central region of the retina immediately ventral of the optic nerve head at $20 \times$ magnification and expressed as cones $/ \mathrm{mm}^{2}$.

IHC analysis of frozen eyecup sections. Procedures have been described previously (Tang et al., 2010). Sections of the central retina (optic nerve was visible) were incubated overnight at $4^{\circ} \mathrm{C}$ with the appropriate primary antibodies and then incubated with the corresponding secondary antibodies and either 4',6-diamidino-2-phenylindole (DAPI) $(2 \mu \mathrm{g} / \mathrm{ml}$ concentration; catalog \#D1306; Invitrogen) or propidium iodide (1 $\mu \mathrm{g} / \mathrm{ml}$ concentration; catalog \#P3566; Invitrogen). Samples were analyzed by confocal microscopy (Leica TCS SP5 AOBS Confocal Microscope System; Leica Microsystems).

Fluorescence quantification within frozen eyecup sections. Age-matched animals were killed, and eyes were prepared and sectioned as described previously (Tang et al., 2010). To minimize differences in the fluorescence signal attributable to the sample preparation procedure, two eyecups from each of the three groups of animals (total of six) were embedded and oriented in a single cassette so that each section contained eyecup samples from all three animal groups. Sections were processed for immunostaining with primary antibodies against RPE65 (PETLET) and S-opsin and imaged by confocal microscopy. For each section, microscopy settings were initially optimized for imaging RPE65 fluorescence within $C 57 B L / 6$ cones and then remained the same for imaging cones from other groups of animals. Single-plane images were acquired through the center of cones that were aligned parallel to the plane of analysis, and images of 10 cones were selected from each group of animals and imported into Adobe Photoshop CS3 software (Adobe Systems) for quantification. A region of analysis (ROA) was drawn around $\mathrm{S}$-opsin fluorescence to calculate the area of the cone outer segment (OS) and was used to measure RPE65 fluorescence contained within. The ROA was then shifted to an immediately adjacent region outside of the cone to measure background fluorescence, which was subtracted from the fluorescence obtained from within the cone. The adjusted value was then divided by the area of the ROA to account for the variability in cone OS size among the different strains/lines of mice and was finally expressed as a fold change of $C 57 B L / 6$ values.

Retinoid delivery. The current protocol for delivering exogenous retinoids to mice was modified from a previously described method (Parker et al., 2009). Retinoids were handled under dim red light. 11-cis-RAL $(0.375 \mathrm{mg} /$ animal $)$ was dissolved in $200 \mu \mathrm{l}$ of vehicle ( $10 \%$ ethanol $/ 10 \%$ bovine serum albumin in $0.9 \% \mathrm{NaCl}$ ), and a single injection was made into the peritoneal cavity of mice ( 6 weeks of age) $12 \mathrm{~h}$ before recording of rod and cone ERGs. The amount of 11-cis-RAL used for this study has been validated previously for evaluating chromophore deficiency in mouse cones (Parker et al., 2009); however, this amount is less than that used previously to rescue cones in $\mathrm{Rpe}^{-1-}$ and $\mathrm{Lrat}^{-/-}$mice (Fan et al., 2008; Zhang et al., 2008). For control, age- and strain/line-matched mice were injected with saline in the peritoneal cavity. After injection, all animals were dark adapted until the onset of recordings.

Measurement of cone and rod function. Procedures were modified from previously described methods (Parker et al., 2009). Mice were anesthetized with xylazine $(20 \mathrm{mg} / \mathrm{kg})$ and ketamine $(80 \mathrm{mg} / \mathrm{kg})$, and pupils were 
dilated with phenylephrine hydrochloride $(2.5 \%)$ and atropine sulfate (1\%). ERG responses were measured using contact lens electrodes placed on both eyes with a drop of methylcellulose. ERGs were recorded with the UTAS E-2000 system (LKC Technologies) using $10 \mathrm{~ms}$ flashes of increasing white-light intensities under scotopic or photopic conditions. Based on previously described retina light loads for mice with and without pigmentation (Lyubarsky et al., 2004), we provided approximately equivalent retinal light stimulus for pigmented $(C 57 B L / 6$ and $B A L B / c \times R p e 65^{-/-}:-3.1,-2.1,-1.1,-0.1$, $0.9,1.9$, and $\left.2.9 \log \mathrm{cd} \cdot \mathrm{s} / \mathrm{m}^{2}\right)$ and albino $(B A L B / c:-3.6,-2.6,-1.6,-0.6,0.4,1.4$, and $\left.2.4 \log \mathrm{cd} \cdot \mathrm{s} / \mathrm{m}^{2}\right)$ mice. A white background light was turned on for 3 min before and maintained for the duration of cone ERG recordings to induce and maintain rod desensitization, which has been shown previously to bleach a large fraction of all three mouse photopigments (Lyubarsky et al., 2004). Similarly, this light source was also adjusted to provide equivalent retina illumination for pigmented $(30 \mathrm{~cd} /$ $\left.\mathrm{m}^{2}\right)$ and albino $\left(10 \mathrm{~cd} / \mathrm{m}^{2}\right)$ mice. Cone b-wave amplitudes were measured from baseline to peak of b-wave. For rod ERG recordings, the a-wave amplitude was measured from baseline to trough of a-wave, and the b-wave amplitude was measured from the trough of a-wave to peak of b-wave. Cone b-waves were plotted with fitted curves using the hyperbolic saturation function, $C / C_{\max }=I / I\left(I+I_{0.5}\right)$. The light sensitivity of the normalized response was defined as $S_{F}=\left[C / C_{\max }\right] / I$ and was simplified to $S_{F}=I_{0.5}$ within the linear range of responses, that is sensitivity was given by the midpoint of the fitted functions.

Statistical analysis. Differences in RPE65 levels between $C 57 B L / 6$ and $B A L B / c, 129 S v$, and each of the cross-strains were tested using ANOVA and Tukey's post hoc tests for individual means. Amounts of RPE65 in eyecups of the various strains/lines of mice were normalized to $\beta$-actin values and expressed as a fold of values obtained for $C 57 B L / 6$ mice. Amounts of RPE65 in $B A L B / c$ and $B A L B / c \times R p e 65^{-/-}$ cones were normalized to values from $C 57 B L / 6$ cones, and the fold change was plotted on a log scale. Two-way ANOVA was used to test for differences in log-transformed values between cones and eyecups across strains. Differences in cone b-wave amplitudes and rod response amplitudes between 11-cis-RAL-treated and untreated mice across the indicated stimulus intensities were also tested using two-way ANOVA. A two-sided $p<0.05$ was considered significant. All analyses were conducted using SAS version 9.1 (SAS Institute).
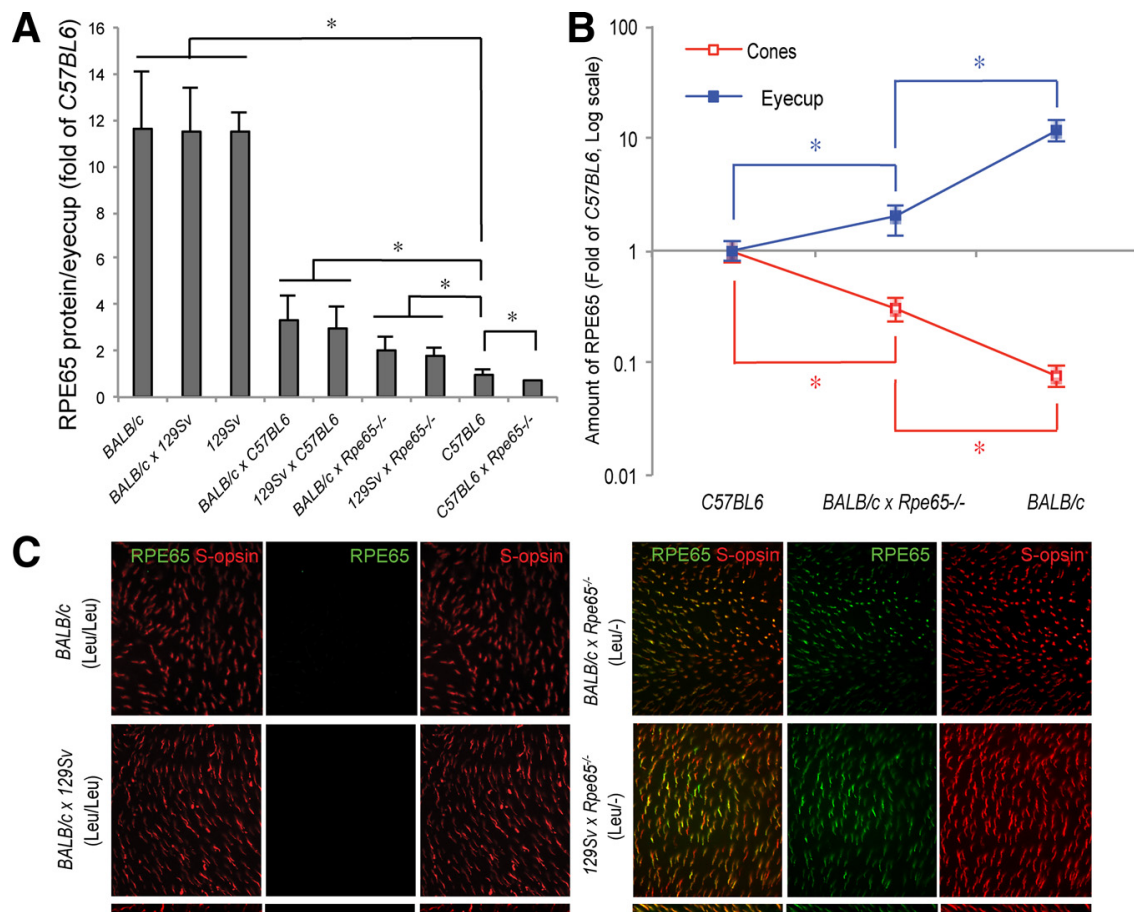

$B A L B / c \times$ Rpe65-/

$B A L B / C$
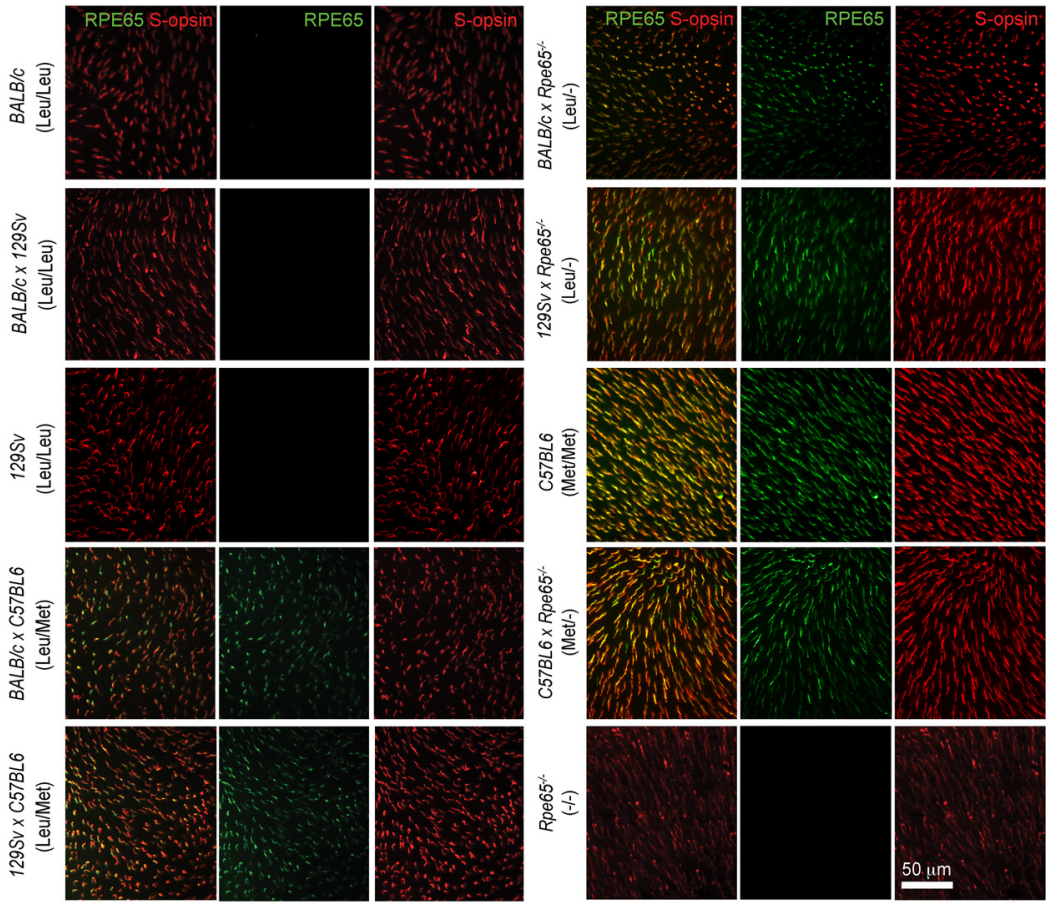

D

Albino C57BL6

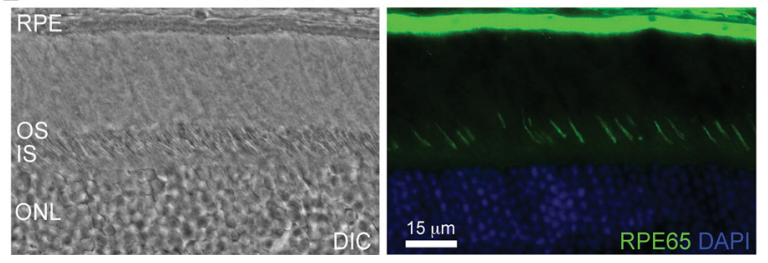

Figure 3. Variation of amount of cone RPE65 among mouse strains/lines. Whole eyecup homogenates were prepared from 4-week-old eyecups. $A$, RPE65 protein per eyecup was quantified from immunoblots for six animals $(n=6)$ from each group using densitometry analysis. Errors are shown as \pm SD. ${ }^{*} p<0.05 . \boldsymbol{B}$, RPE65 immunofluorescence was quantified for cones $(n=10)$ from each indicated group, background fluorescence was subtracted, and values are plotted on a logarithmic scale in red as fold of amount quantified for C57BL/6 cones (C57BL/6 $=1.00 \pm 0.22 ; B A L B / C \times R p e 65^{-/}=0.31 \pm 0.07 ; B A L B / C=0.08 \pm 0.02$ ) with the amount of RPE65 per eyecup calculated from $\boldsymbol{A}$ displayed in blue. ${ }^{*} p<0.001$. Errors are represented as \pm SD. $C$, Retinas (2 week-old Rpe65 ${ }^{-1-}$ mice; 4-week-old for all other strains/lines) from each group are costained for RPE65 and S-opsin and flat mounted for analysis. Status of the amino acid at position 450 of RPE65 is indicated for each group. Images are taken from the central region of the retina immediately ventral to the optic nerve head. D, RPE65 is detected within cones of albino C57BL/6 mice. ONL, Outer nuclear layer.

\section{Results}

\section{RPE65 protein localization within the $C 57 B L / 6$ mouse cone}

To investigate the RPE65 staining within mouse cones, frozen sections were prepared from eyecups of 4 -week-old $C 57 B L / 6$ and $B A L B / c$ and 2-week-old Rpe65 $5^{-1-}$ mice. The PETLET polyclonal antibody has been shown to have a high specificity for mouse RPE65 (Redmond and Hamel, 2000). The RPE of wild-type mice served as a positive control, and RPE65 staining was detected within the RPE of $C 57 B L / 6$ and $B A L B / c$ eyecups but not in Rpe $65^{-/-}$eyecups (Fig. 1A). RPE65 staining was detected in $C 57 B L / 6$ mouse cones (Fig. $1 A$, top) but not in the cones of $B A L B / c$ (middle) or Rpe65 $5^{-/-}$(bottom) mice. Costaining with an antibody for S-opsin confirmed that these cells are cones. $z$-series images were collected from $C 57 B L / 6$ cones, 
A

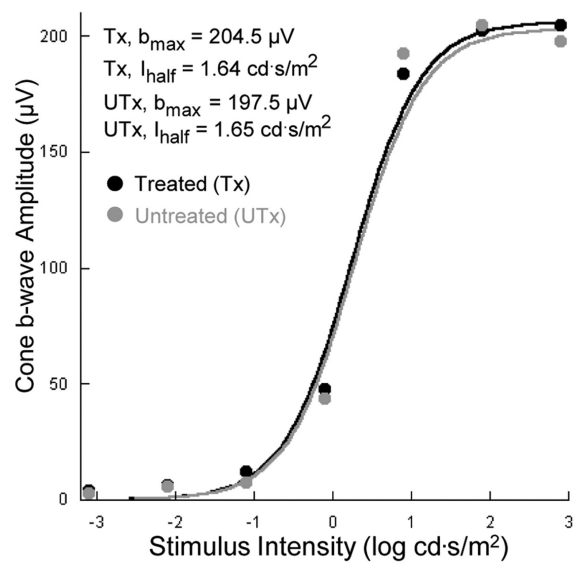

B

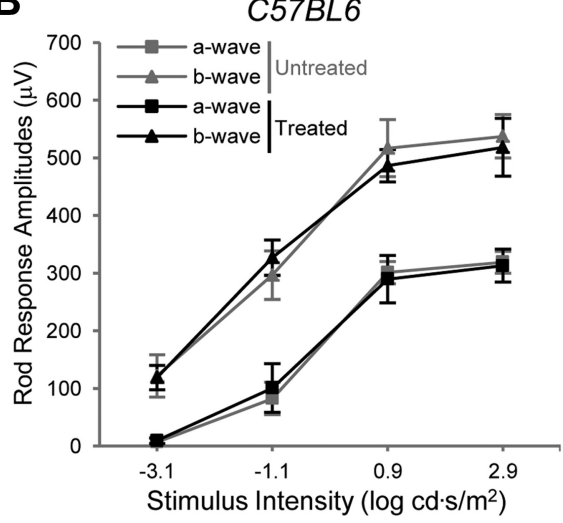

$B A L B / c \times$ Rpe $65^{-1}$

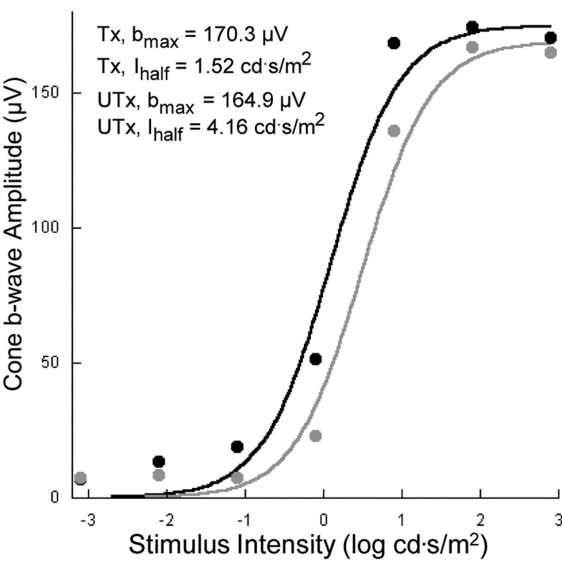

BALB/c $\times$ Rpe $65^{-/}$

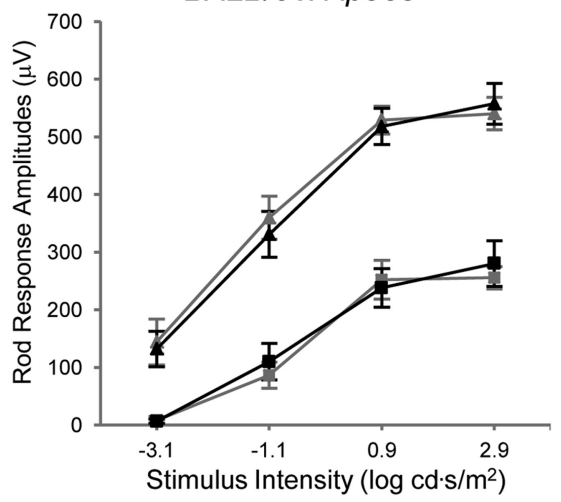

\section{$B A L B / C$}

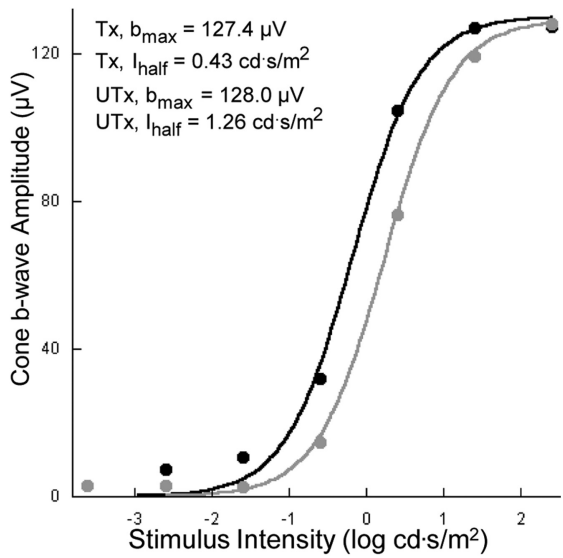

$B A L B / C$

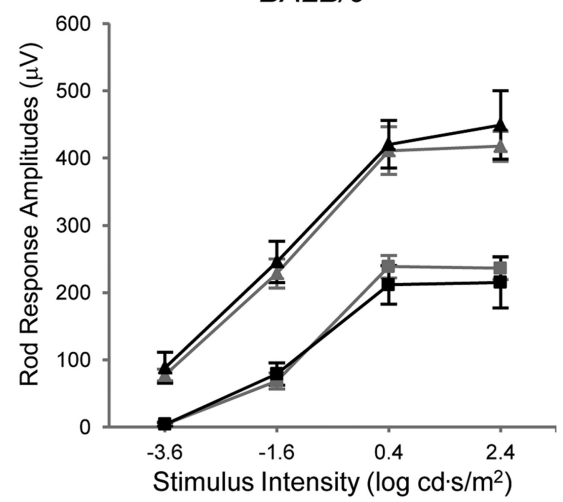

C

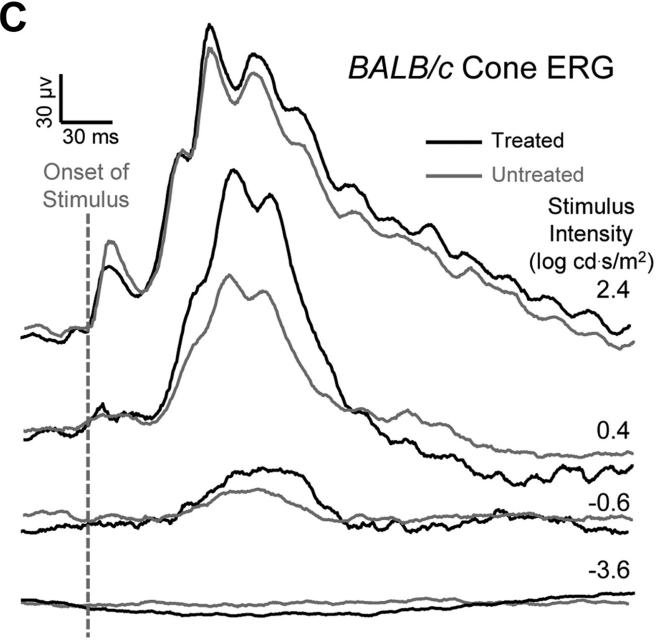

D

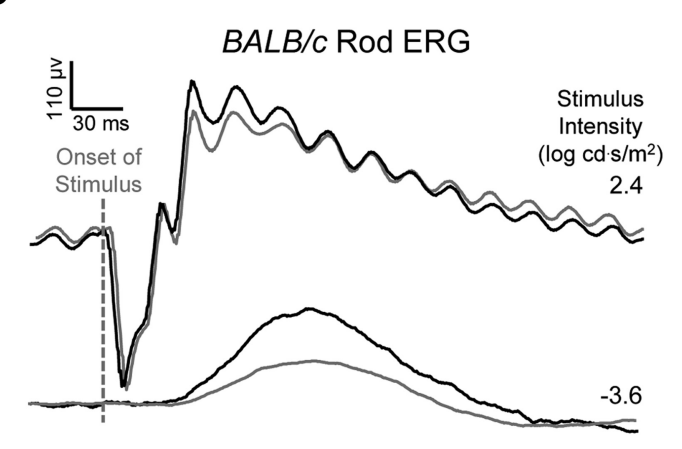

Figure 4. Effects of exogenous 11-cis-RAL on cone and rod function. Six-week-old animals ( $n=5$ per group) were intraperitoneally injected with $0.375 \mathrm{mg}$ of 11 -cis-RAL (treated) or saline (untreated) $12 \mathrm{~h}$ before ERG recordings. $A$, Summaries of cone responses are shown for mice exhibiting high $(C 57 B L / 6)$, intermediate $\left(B A L B / C \times R\right.$ Re $\left.65^{-1-}\right)$, and undetectable $(B A L B / C)$ levels of RPE65 within cones (Fig. 3B). An approximate threefold increase in the sensitivity of cone b-waves is observed for $B A L B / C$ and $B A L B / C \times R$ Re $65^{-/-}$mice. $B$, No significant differences were observed in rod responses between treated and untreated mice. $\boldsymbol{C}, \boldsymbol{D}$, Sample cone $(\boldsymbol{C})$ and $\operatorname{rod}(\boldsymbol{D})$ ERG traces are shown for treated and untreated $B A L B / C$ mice. Errors are represented as $\pm S D$.

showing that the majority of RPE65 was localized at the base of the OS region with punctate particles appearing apically (Fig. $1 B)$. Because the isomerase of the intraretinal visual cycle has been implicated to exist within Müller cells, we evaluated whether RPE65 is also present within this cell type by costaining C57BL/6 eyecup sections for RPE65 and glutamine synthetase, a glial marker. Our results indicate that RPE65 is absent from Müller cells and is localized only to the RPE and cones within the C57BL/6 mouse eyecup (Fig. 1C). To determine whether RPE65 was also present within the inner segment (IS) region of the cone, counterstaining with propidium iodide (Fig. $1 D$ ) or FITC-conjugated peanut agglutinin (Fig. $1 E$ ) was performed. These results suggest that RPE65 was not localized within the IS.

The specificity of the antibody is of importance for detecting RPE65 in cones. Using the 8B11 monoclonal anti-RPE65 antibody, staining within cones was not detected in flat-mounted 
4-week-old C57BL/6 mouse retina (Fig. $2 A$ ), in agreement with results previously reported with this antibody by Hemati et al. (2005). Additional confirmation was provided through similar analysis of frozen $C 57 B L / 6$ eyecup sections, with $8 \mathrm{~B} 11$ antibody staining detected only within the RPE (Fig. $2 B$ ). We conclude that the PETLET antibody exhibits both higher affinity and greater specificity for RPE65 than the 8B11 antibody, with immunoblot analysis providing additional support (Fig. 2C). Because the amount of RPE65 protein within cones is minute compared with that found within the RPE, the lower affinity and specificity of the 8B11 antibody may account for the discrepancies in the previously reported data (Hemati et al., 2005), thereby settling this controversy.

\section{Variation of total RPE65 protein in eyecup across mouse strains/lines}

The quantity of RPE65 protein within the whole eyecup has been shown previously to vary among mouse strains/lines (Lyubarsky et al., 2005), a feature that may be related to the status of the amino acid at position 450 of the protein (Wenzel et al., 2001). $B A L B / c$ mice possess a variant of the Rpe65 gene that encodes for leucine at position 450 (Leu450) (Wenzel et al., 2001) and exhibit an $\sim 11$-fold greater amount of the protein per eyecup compared with C57BL/6 mice (Lyubarsky et al., 2005), which encode for methionine (Met450) (Wenzel et al., 2001). Previous studies have shown that the amount of RPE65 in the RPE can be varied by crossing $B A L B / c$ mice with $C 57 B L / 6$ and $R p e 65^{-/-}$mice (Lyubarsky et al., 2005). To determine whether the absence of RPE65 protein from $B A L B / c$ cones was related to the higher amount of RPE65 in the RPE, we generated mice with varying amounts of RPE65 by crossing the different lines. Performing similar crosses with $129 S v$ mice served to confirm our results, because these mice also express the Leu450 variant of the Rpe65 gene (Wenzel et al., 2001). Because the amount of RPE65 protein that is present within cones is negligible compared with that in the RPE, we assume that a change in the amount of RPE65 protein per eyecup reflects a change in the RPE. Quantification of RPE65 within the eyecups using densitometry analysis of the various strains and crosses is summarized in Figure 3. Relative to the amount of RPE65 protein present in $C 57 B L / 6$ eyecups, animals homozygous for the Leu450 variant of Rpe 65 gene $(B A L B / c, B A L B / c \times 129 S v$, $129 S v$ ) exhibited the greatest amount per eyecup of all the mouse strains/lines evaluated. Those that are mixed Leu/Met 450 variants $(B A L B / c \times C 57 B L / 6,129 S v \times C 57 B L / 6)$ or are heterozygous for the Leu450 variant of the gene $\left(B A L B / c \times R p e 65^{-/-}, 129 S v \times\right.$ Rpe $65^{-/-}$) exhibited an intermediate amount. Finally, animals that are heterozygous for the Met 450 variant $(C 57 B L / 6 \times$ Rpe $65^{-/-}$) exhibited the lowest amount. Our results from $C 57 B L / 6, B A L B / c$, and the corresponding crossed mice are in agreement with those published previously (Lyubarsky et al., 2005).

\section{Inverse relationship of RPE65 quantities within the RPE and cones}

Mouse strains/lines that exhibit greater amounts of RPE65 protein per eyecup also have been shown to exhibit a faster rate of rod photopigment regeneration (Lyubarsky et al., 2005), suggesting that the robustness of the RPE-retinal visual cycle is dependent on the amount of RPE65 protein in the RPE. We observed a correlation between the levels of RPE65 expressed in the RPE and in cones; that is, mouse strains with a robust RPE-retinal visual cycle are less likely to have RPE65 expressed within cones. The lack of detectable RPE65 protein within $B A L B / c$ cones (Fig. $1 A$ )
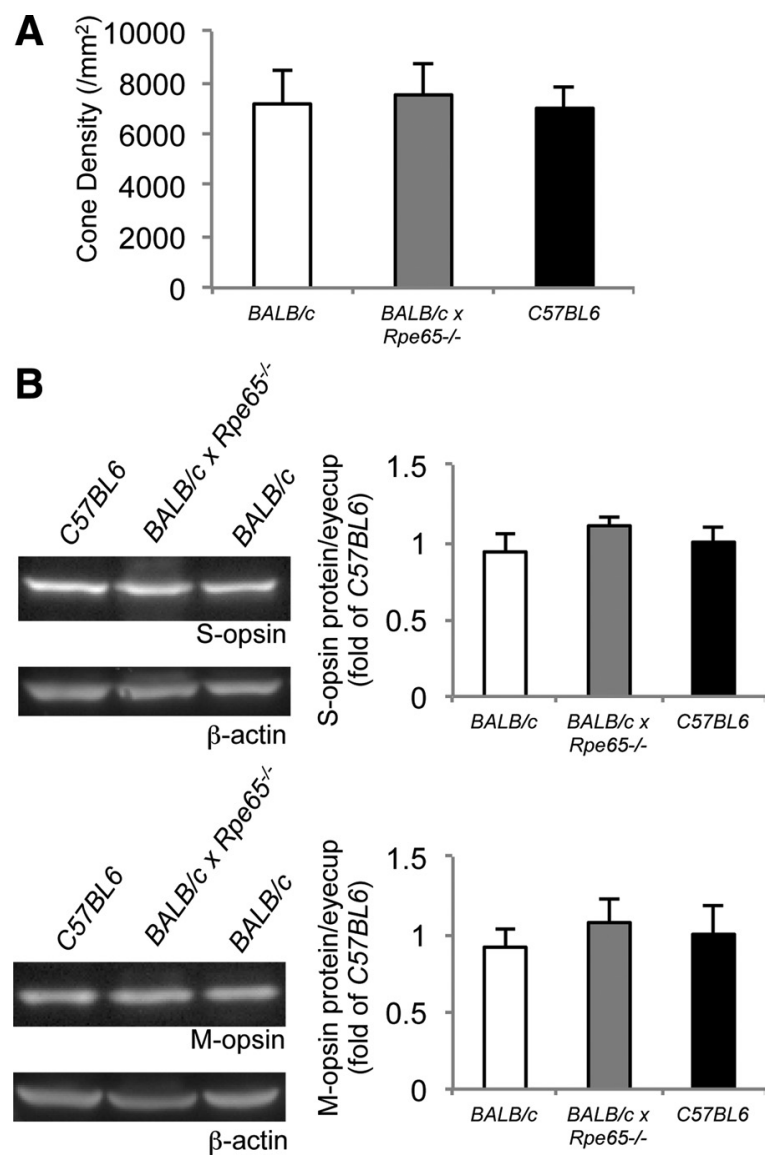

Figure 5. Comparable cone levels in retinas of $C 57 B L / 6, B A L B / C \times R p e 65^{-/-}$, and $B A L B / C$ mice. $A$, Cone densities measured by $S$-opsin staining from the central region of $B A L B / C(p=$ $0.82 ; n=5)$ and $B A L B / C \times R p e 65^{-1-}(p=0.46 ; n=5)$ retina flat mounts did not differ significantly from that of $C 57 B L / 6$ mice $(n=5)$. $B$, This was further confirmed from quantification of both $\mathrm{M}$ - and $\mathrm{S}$-cone opsin proteins per eyecup using immunoblot analysis, indicating the amounts of both $\mathrm{M}$ - and S-opsins from BALB/C $(n=5)$ and $B A L B / C \times R p e 65^{-/-}(n=5)$ eyecups did not differ significantly from that of $C 57 B L / 6(n=5)$ eyecups after normalization to $\beta$-actin. Errors are represented as \pm SD.

may be related to its high amount in the RPE, which could enable the RPE-retinal visual cycle to meet the chromophore demands of cones in bright light. The RPE-retinal visual cycle in C57BL/6 mice is less robust because of an 11-fold lower amount of RPE65 (Fig. 3A); therefore, its cones may be more dependent on the intraretinal visual cycle for chromophore and express a greater amount of proteins involved in that pathway such as RPE65. To further investigate, retinas from each mouse strain/line were flat mounted and costained for RPE65 and the S-opsin as a marker for cone OS. Images of flat-mounted retina from the different crosses are shown in Figure $3 C$. Strains with the greatest amount of RPE65 protein $(B A L B / c, B A L B / c \times 129 S v, 129 S v)$ did not exhibit detectable RPE65 staining within cones. When the amount of RPE65 was reduced to intermediate $(B A L B / c \times C 57 B L / 6$, $129 S v \times C 57 B L / 6, B A L B / c \times R p e 65^{-/-}$, and $\left.129 S v \times R p e 65^{-/-}\right)$ or lower $\left(C 57 B L / 6 \times R p e 65^{-/-}\right)$levels relative to that observed in $C 57 B L / 6$ mice, RPE65 staining was detected within cones. The lack of RPE65 within $B A L B / c$ cones was not related to the lack of pigmentation, because pigmented $129 S v$ mice showed a similar result and RPE65 was detected in cones of albino C57BL/6 mice (Fig. 3D). Furthermore, the absence of RPE65 in cones is not believed to be related to the exclusive expression of the Leu450 variant by $B A L B / c$ and $129 S v$ mice, because $B A L B / c \times R p e 65^{-/-}$ 
and $129 \mathrm{~Sv} \times \mathrm{Rpe} 65^{-/-}$mice also exclusively express this variant, yet the protein is present within cones (Fig. 3C). We were able to compare the relative amount of RPE65 protein among cones of $B A L B / c, B A L B / c \times R p e 65^{-/-}$, and $C 57 B L / 6$ mice through quantifying fluorescence from RPE65 staining in eyecup sections prepared from litters that were born on the same day. Displayed as a fold of fluorescence detected in C57BL/6 cones ( $1 \pm 0.22), B A L B /$ $c \times R$ pe $65^{-/-}$cones exhibited intermediate levels $(0.31 \pm 0.07)$, whereas $B A L B / c$ cones exhibited the lowest levels $(0.08 \pm 0.02)$. This is opposite of that observed from quantifying RPE65 protein per eyecup (Fig. $3 A$ ), in which $B A L B / c \times R p e 65^{-/-}$and $B A L B / c$ eyecups exhibited $\sim 2$ and 11 times more of the protein than $C 57 B L / 6$ eyecups, respectively. The inverse relationship between the quantities of RPE65 in the two cell types among the three groups of animals analyzed is summarized in Figure $3 B$. The mechanism regulating this observation is currently unclear. It is possible that 11-cis-RAL could act as an inhibitory signal for Rpe65 transcription in cones. Additional studies will be required to understand how the expression of RPE65 within the RPE may affect the expression of the protein within cones.

\section{Enhanced sensitivity after chromophore supplementation in cones lacking RPE65}

To investigate whether the quantity of cone RPE65 had an effect on the ability of cones to regenerate photopigment under brightlight conditions, we intraperitoneally injected 11-cis-RAL into agematched (6-week-old) C57BL/6, BALB/c $\times R$ Re $65^{-/-}$, and BALB/c mice (treated group) $12 \mathrm{~h}$ before recording rod (scotopic) and cone (photopic) ERGs using white-light stimuli and compared them with corresponding lines of mice receiving saline injections (untreated group). Both the stimuli and the background light were adjusted to provide an equivalent amount of retina illumination in pigmented $\left(C 57 B L / 6\right.$ and $\left.B A L B / c \times R p e 65^{-/-}\right)$and albino $(B A L B / c)$ mice, as described in previous studies (Lyubarsky et al., 2004). In both $B A L B / c$ and $B A L B / c \times R p e 65^{-/-}$mice, 11-cis-RAL treatment resulted in significant $(p<0.05)$ increases in the cone response, prompting additional evaluation of cone sensitivity. An approximate threefold increase in the sensitivity of the cone b-wave in the presence of a background light that is expected to bleach a large fraction of photopigments was observed in both strains/lines of mice, with negligible change in the saturated amplitude (Fig. 4A). Because the bleaching rate constants must be the equivalent in treated and untreated mice of the same strain/line, the only plausible explanation for the increased sensitivity is that exogenous 11-cisRAL provides an effective source of chromophore that exceeds the native sources by threefold for cones in $B A L B / c$ and $B A L B / c \times$ Rpe $65^{-/-}$mice but not in C57BL/6 mice.

Rod responses (both a- and b-waves) were not altered significantly after 11-cis-RAL treatment in any of the three strains/lines of mice (Fig. $4 \mathrm{~B}$ ). The density of cones within the retina and total cone opsin levels were evaluated, revealing no significant difference in the number of cones among the three groups of mice (Fig. 5 ). Because white light was used as a stimulus, we were unable to differentiate the relative contributions of $\mathrm{M}$ - and S-opsin pigments to the increase in $B A L B / c \times R p e 65^{-/-}$and $B A L B / c$ cone sensitivity after 11-cis-RAL administration. We also observed that the overall b-wave amplitudes of cone ERGs recorded from $B A L B / c$ mice were lower than those recorded from the other two groups; however, this may be an effect of a possible inherent difference in the characteristics of bipolar cells between various strains of mice and not attributable to differences in the efficiency of cone photopigment regeneration. These data show that chromophore supplementation uniquely enhanced the sensitivity of cones with decreased or undetectable amounts of RPE65 relative to C57BL/6 cones. Because $C 57 B L / 6$ mice have much less RPE65 overall in the retina, we conclude that the RPE65 expressed in their cones is effective for providing 11-cis-RAL, and thus cone RPE65 may be essential for maintaining full chromophore levels under bright-light conditions.

\section{Discussion}

Absence of RPE65 immunostaining within $B A L B / c$ and $129 S v$ mouse cones

The existence of RPE65 within cones has been a controversial topic, because discrepancies between the findings of various groups have remained unresolved. Using the polyclonal PETLET anti-RPE65 antibody, Znoiko et al. (2002) reported the detection of staining within the flat-mounted retinas of mice, including $C 57 B L / 6, B A L B / c$, and $129 S v$ strains. Using the same antibody, our IHC results show that positive staining is observed only within $C 57 B L / 6$ cones. After extensive evaluation of $B A L B / c$ and $129 S v$ mouse eyecups, we have been unable to detect the presence of RPE65 protein within cones of these two strains. This is an important clarification, because the presence of RPE65 within mouse cones differs across strains and is further explored in the current study. Our results with the 8B11 antibody suggest that its lower affinity and specificity resulted in the lack of detection of RPE65 within cones, consistent with the previously reported data (Hemati et al., 2005).

\section{Involvement of RPE65 within the intraretinal visual cycle}

The lack of RPE65 staining observed within $B A L B / c$ and $129 S v$ mouse cones prompted additional investigation. The robustness of the RPE-retinal visual cycle for recycling chromophore is dependent on the amount of RPE65 present within the RPE (Wenzel et al., 2001). The rod photopigment regeneration rate of $B A L B / c$ mice is more than three times faster than that of $C 57 B L / 6$ mice because of an 11-fold increase in the amount of RPE65 protein within the RPE (Wenzel et al., 2001; Lyubarsky et al., 2005); however, RPE65 was undetectable in cones of the $B A L B / c$ retina (Fig. $1 A$, middle). The appearance of RPE65 within cones (Fig. 3C) correlated only with animals that expressed reduced amounts of RPE65 within the RPE relative to $B A L B / c$ and $129 S v$ mice (Fig. $3 A$ ). Functionally, the ability of cones to regenerate photopigment efficiently in bright-light conditions appears to be dependent on the amount of cone RPE65 that is present (Fig. $4 A$ ). These results suggest that cone RPE65 may be involved in the intraretinal visual cycle to promote the regeneration of cone photopigment in animals in which the RPE-retinal visual cycle cannot meet its chromophore demands. At the present time, we cannot rule out the possibility that the appearance of RPE65 within cones is related to the $C 57 B L / 6$ genotype. Developing and evaluating an Rpe65 conditional knock-out mouse model from the $C 57 B L / 6$ background is an essential step to verifying our current findings.

\section{Possible role of RPE65 within cones}

Additional examination of the pattern of RPE65 localization within the $C 57 B L / 6$ mouse cone may provide insights into its specific function. RPE65 protein assumes a characteristic distribution pattern within the OS, with the protein being most abundant at the interface with the IS and a punctate distribution appearing apically. Subcellular characterization will require techniques that offer greater resolution such as immunoelectron microscopy. 
Within the RPE, RPE65 acts as the isomerohydrolase in the RPE-retinal visual cycle to convert all-trans retinoids to 11-cisforms (Moiseyev et al., 2005). All-trans retinyl esters (RE) serve as substrates for RPE65 (Moiseyev et al., 2003), which are generated through the transfer of a fatty acyl group from phosphatidylcholine to all-trans ROL by enzymes such as lecithin:retinol acyltransferase (LRAT) and acyl-CoA:retinol acyltransferase (ARAT) (Saari and Bredberg, 1989; Batten et al., 2004; Kaschula et al., 2006; Muniz et al., 2006, 2009; Travis et al., 2007). LRAT has been identified as the major RE synthase within the RPE to support the RPE-retinal visual cycle (Saari and Bredberg, 1989; Batten et al., 2004). Although ARAT has also been shown to be active within the RPE (Kaschula et al., 2006), evidence suggests that it is the major RE synthase within the Müller cell (Muniz et al., 2006) to support the intraretinal visual cycle (Muniz et al., 2009). Because neither LRAT nor ARAT have been identified to exist within cones, cone RPE65 lacks the necessary substrate to function as an isomerohydrolase. Interestingly, studies performed in an immortalized cone photoreceptor cell line (661W) have suggested the existence of a novel mechanism for synthesizing RE that is both LRAT and ARAT independent (Kanan et al., 2008) and may potentially provide a source of RE for cone RPE65. Additional work is needed to clarify the mechanism for this pathway as well as to evaluate whether these observations made in vitro reflect a process occurring in vivo.

How can cone RPE65 contribute to photopigment formation? Within the intraretinal visual cycle, Müller cells have been implicated to isomerize all-trans ROL to 11-cis-ROL (Das et al., 1992), which is then delivered to the cone by the inter-photoreceptor retinoid binding protein (Parker et al., 2009). There are reports suggesting that the cell body region of the cone receives incoming 11-cis-ROL from Müller cells, because morphological data indicate that Müller cell processes make multiple associations with the cones at this region (Sarantis and Mobbs, 1992; Wang et al., 2009), and in vitro data show that cones can use 11-cis-RAL for photopigment production only when it is delivered through this site (Jin et al., 1994). The ability of cones to oxidize 11-cis-ROL to 11-cis-RAL may be attributable to either an unidentified retinol dehydrogenase (RDH) enzyme or an uncharacterized function of a known RDH (Parker and Crouch, 2010). The characteristic appearance of RPE65 distribution within the cone OS suggests that RPE65 may be acting as a binding protein to shuttle 11-cisROL from the IS to the OS for oxidation to 11-cis-RAL. This role may be essential for satisfying the high demands for cone function in bright-light conditions as newly synthesized chromophore is placed immediately adjacent to opsins for photopigment formation. Analysis of the native crystal structure (Kiser et al., 2009) and identification of proteins that are coeluted through affinity purification experiments (Hemati et al., 2005) suggest that RPE65 is capable of interacting with other proteins, such as RDHs. Therefore, RPE65 may further promote oxidation of its bound 11-cis-ROL with an unidentified RDH to generate 11-cis-RAL. Additional experiments are necessary to address the specific function of RPE65 within cones.

The existence of RPE65 protein within cones has been debated, but these data settle this controversy. An antibody with high sensitivity and specificity for the protein is required to detect these relatively low amounts within cones, particularly with the high concentrations present within the RPE. RPE65 is not present (or at least not detectable) within cones of all strains/lines of mice; however, our data suggest that its presence greatly enhances the function of cones in bright-light conditions. This current study represents the first in vivo evidence implicating RPE65 to be involved in the cone-specific intraretinal visual cycle.

\section{References}

Ala-Laurila P, Cornwall MC, Crouch RK, Kono M (2009) The action of 11-cis retinol on cone opsins and intact cone photoreceptors. J Biol Chem 284:16492-16500.

Batten ML, Imanishi Y, Maeda T, Tu DC, Moise AR, Bronson D, Possin D, Van Gelder RN, Baehr W, Palczewski K (2004) Lecithin-retinol acyltransferase is essential for accumulation of all-trans-retinyl esters in the eye and in the liver. J Biol Chem 279:10422-10432.

Baylor DA, Lamb TD, Yau KW (1979) The membrane current of single rod outer segments. J Physiol 288:589-611.

Baylor DA, Nunn BJ, Schnapf JL (1984) The photocurrent, noise and spectral sensitivity of rods of the monkey Macaca fascicularis. J Physiol 357:575-607.

Das SR, Bhardwaj N, Kjeldbye H, Gouras P (1992) Muller cells of chicken retina synthesize 11-cis-retinol. Biochem J 285:907-913.

Fan J, Rohrer B, Frederick JM, Baehr W, Crouch RK (2008) RPE65-/- and LRAT - / - mice: comparable models of Leber Congenital Amaurosis. Invest Ophthalmol Vis Sci 49:2384-2389.

Hemati N, Feathers KL, Chrispell JD, Reed DM, Carlson TJ, Thompson DA (2005) RPE65 surface epitopes, protein interactions, and expression in rod- and cone-dominant species. Mol Vis 11:1151-1165.

Jin J, Jones GJ, Cornwall MC (1994) Movement of retinal along cone and rod photoreceptors. Vis Neurosci 11:389-399.

Jin M, Li S, Moghrabi WN, Sun H, Travis GH (2005) Rpe65 is the retinoid isomerase in bovine retinal pigment epithelium. Cell 122:449-459.

Jones GJ, Crouch RK, Wiggert B, Cornwall MC, Chader GJ (1989) Retinoid requirements for recovery of sensitivity after visual-pigment bleaching in isolated photoreceptors. Proc Natl Acad Sci U S A 86:9606-9610.

Kanan Y, Kasus-Jacobi A, Moiseyev G, Sawyer K, Ma JX, Al-Ubaidi MR (2008) Retinoid processing in cone and Müller cell lines. Exp Eye Res 86:344-354.

Kaschula CH, Jin MH, Desmond-Smith NS, Travis GH (2006) Acyl CoA: retinol acyltransferase (ARAT) activity is present in bovine retinal pigment epithelium. Exp Eye Res 82:111-121.

Kiser PD, Golczak M, Lodowski DT, Chance MR, Palczewski K (2009) Crystal structure of native RPE65, the retinoid isomerase of the visual cycle. Proc Natl Acad Sci U S A 106:17325-17330.

Kono M, Goletz PW, Crouch RK (2008) 11-cis and all-trans retinols can activate rod opsin: rational design of the visual cycle. Biochemistry 47:7567-7571.

Lyubarsky AL, Daniele LL, Pugh EN Jr (2004) From candelas to photoisomerizations in the mouse eye by rhodopsin bleaching in situ and the light-rearing dependence of the major components of the mouse ERG. Vision Res 44:3235-3251.

Lyubarsky AL, Savchenko AB, Morocco SB, Daniele LL, Redmond TM, Pugh EN Jr (2005) Mole quantity of RPE65 and its productivity in the generation of 11-cis retinal from retinyl esters in the living mouse eye. Biochemistry 44:9880-9888.

Ma J, Xu L, Othersen DK, Redmond TM, Crouch RK (1998) Cloning and localization of RPE65 mRNA in salamander cone photoreceptor cells. Biochim Biophys Acta 1443:255-261.

Mata NL, Radu RA, Clemmons RC, Travis GH (2002) Isomerization and oxidation of vitamin A in cone-dominant retinas: a novel pathway for visual-pigment regeneration in daylight. Neuron 36:69-80.

Moiseyev G, Crouch RK, Goletz P, Oatis J Jr, Redmond TM, Ma JX (2003) Retinyl esters are the substrate for isomerohydrolase. Biochemistry 42:2229-2238.

Moiseyev G, Chen Y, Takahashi Y, Wu BX, Ma JX (2005) RPE65 is the isomerohydrolase in the retinoid visual cycle. Proc Natl Acad Sci U S A 102:12413-12418.

Muniz A, Villazana-Espinoza ET, Thackeray B, Tsin AT (2006) 11-cis-AcylCoA:retinol O-acyltransferase activity in the primary culture of chicken Müller cells. Biochemistry 45:12265-12273.

Muniz A, Villazana-Espinoza ET, Hatch AL, Trevino SG, Allen DM, Tsin AT (2007) A novel cone visual cycle in the cone-dominant retina. Exp Eye Res 85:175-184.

Muniz A, Betts BS, Trevino AR, Buddavarapu K, Roman R, Ma JX, Tsin AT (2009) Evidence for two retinoid cycles in the cone-dominant chicken eye. Biochemistry 48:6854-6863.

Parker RO, Crouch RK (2010) Retinol dehydrogenases (RDHs) in the visual cycle. Exp Eye Res 91:788-792.

Parker RO, Fan J, Nickerson JM, Liou GI, Crouch RK (2009) Normal cone 
function requires the interphotoreceptor retinoid binding protein. J Neurosci 29:4616-4621.

Perry RJ, McNaughton PA (1991) Response properties of cones from the retina of the tiger salamander. J Physiol 433:561-587.

Redmond TM, Hamel CP (2000) Genetic analysis of RPE65: from human disease to mouse model. Methods Enzymol 316:705-724.

Redmond TM, Poliakov E, Yu S, Tsai JY, Lu Z, Gentleman S (2005) Mutation of key residues of Rpe65 abolishes its enzymatic role as isomerohydrolase in the visual cycle. Proc Natl Acad Sci U S A 102:13658-13663.

Saari JC, Bredberg DL (1989) Lecithin:retinol acyltransferase in retinal pigment epithelial microsomes. J Biol Chem 264:8636-8640.

Sarantis M, Mobbs P (1992) The spatial relationship between Müller cell processes and the photoreceptor output synapse. Brain Res 584:299-304.

Schnapf JL, Nunn BJ, Meister M, Baylor DA (1990) Visual transduction in cones of the monkey Macaca fascicularis. J Physiol 427:681-713.

Seeliger MW, Grimm C, Ståhlberg F, Friedburg C, Jaissle G, Zrenner E, Guo H, Remé CE, Humphries P, Hofmann F, Biel M, Fariss RN, Redmond TM, Wenzel A (2001) New views on Rpe65 deficiency: the rod system is the source of vision in a mouse model of Leber Congenital Amaurosis. Nat Genet 29:70-74.

Tang PH, Fan J, Goletz PW, Wheless L, Crouch RK (2010) Effective and sustained delivery of hydrophobic retinoids to photoreceptors. Invest Ophthalmol Vis Sci 51:5958-5964.

Travis GH, Golczak M, Moise AR, Palczewski K (2007) Diseases caused by defects in the visual cycle: retinoids as potential therapeutic agents. Annu Rev Pharmacol Toxicol 47:469-512.

Wang JS, Kefalov VJ (2009) An alternative pathway mediates the mouse and human cone visual cycle. Curr Biol 19:1665-1669.

Wang JS, Kefalov VJ (2011) The cone-specific visual cycle. Prog Retin Eye Res 30:115-128.

Wang JS, Estevez ME, Cornwall MC, Kefalov VJ (2009) Intra-retinal visual cycle required for rapid and complete cone dark adaptation. Nat Neurosci 12:295-302.

Wenzel A, Reme CE, Williams TP, Hafezi F, Grimm C (2001) The Rpe65 Leu450Met variation increases retinal resistance against light-induced degeneration by slowing rhodopsin regeneration. J Neurosci 21:53-58.

Zhang H, Fan J, Li S, Karan S, Rohrer B, Palczewski K, Frederick JM, Crouch RK, Baehr W (2008) Trafficking of membrane-associated proteins to cone photoreceptor outer segments requires the chromophore 11-cisretinal. J Neurosci 28:4008-4014.

Znoiko SL, Crouch RK, Moiseyev G, Ma JX (2002) Identification of the RPE65 protein in mammalian cone photoreceptors. Invest Ophthalmol Vis Sci 43:1604-1609. 